\title{
Fighting Against COVID-19 at the Community Level - Wuhan City, Hubei Province, China, 2020
}

\author{
Canyou Zhang ${ }^{1,2}$; George F. Gao'; Zhifeng Wang ${ }^{2}$; Hao Wu ${ }^{3, *}$
}

Since December 2019, an increasing number of cases of pneumonia with unknown etiology (PUE)_later named as coronavirus disease 2019 (COVID-19) by the World Health Organization (WHO) — was identified in Wuhan (1-3), a large city of 11 million people in central China, and the basic reproductive number $\left(\mathrm{R}_{0}\right)$ was estimated to be 2.2 (95\% CI: 1.4 to 3.9) (4). On March 11, 2020, the WHO formally announced COVID-19 a worldwide pandemic based on the alarming levels of spread and severity and by the alarming levels of inaction (5). Although there is neither a vaccine nor specific medication available, the pandemic was well contained in China by the strict introduction of nonpharmacological interventions (NPIs) (6).

To accomplish this, community-level control and prevention that took measures to "turn off the tap" of COVID-19 cases was key. Through in depth grid management and extensive community mobilization, communities were able to achieve earlier detection, earlier reporting, and reduced risk of transmission. Our field experiences in Wuhan are summarized here, and the accomplishments were also aided by the mobilization of approximately 42,000 health-care workers (HCWs) from all over China that provided an emergency response and addressed major logistical challenges.

Wuhan City has four levels of administrative division including city level, district level, street level, and community level. Community is the most fundamental unit of the society and also the critical battle field to control increasing COVID-19 cases. On February 6, the National Health Commission of China sent a community work group to Wuhan to guide COVID-19 control in communities, and this group consisted of 13 community health specialists and 13 experts from China CDC, which were then made into pairs for the 13 districts of Wuhan.

\section{Community-level Lockdown}

At the early stages of the pandemic, there were not enough beds in hospitals for patients and insufficient nucleic acid test capacity for diagnosis, so many patients later confirmed with COVID-19 were still mobile in their communities and visited hospitals and supermarkets. As a first measure to address the uncontrolled spread in face of logistical problems ("calming the water of the lake"), we recommended on February 9, 2020 in Jiang'an District to close neighborhoods immediately, and we called on residents to stay at home and avoid going outside as much as possible. This policy was extended to the whole city on February 11, and daily necessities and required medical supplies were a priority to be replenished.

\section{City Grid Management and Division of Work}

A community was divided into several grid boxes that usually contain 300 to 800 households. The number of households in each grid box depended on the status of neighborhoods it contains: small grid boxes consisted of old neighborhoods without property management, and large grid boxes consisted of neighborhoods with good property management. Wuhan had more than 13,800 grid boxes, and in general, each community had 5 to 7 officers, 5 to 8 coordinators, and several grid workers. The workforce was critically inadequate during the epidemic, so we recommended arranging recruitment of volunteers including government officers, enterprise employees, etc., to participate in grid management. During this process, HCWs from Community Health Service Center played an important role in grid management (Figure 1).

Persuasion at the entrance: Property management Staff and/or volunteers were on duty at the entrance to the neighborhoods to measure body temperature, check identification of all persons who went in and out of the neighborhood, and persuade them to go out infrequently and maintain social distancing. Moreover, they disseminated health promotion materials of COVID-19 and helped with the logistics for managing the residents, and some communities supplied free face 


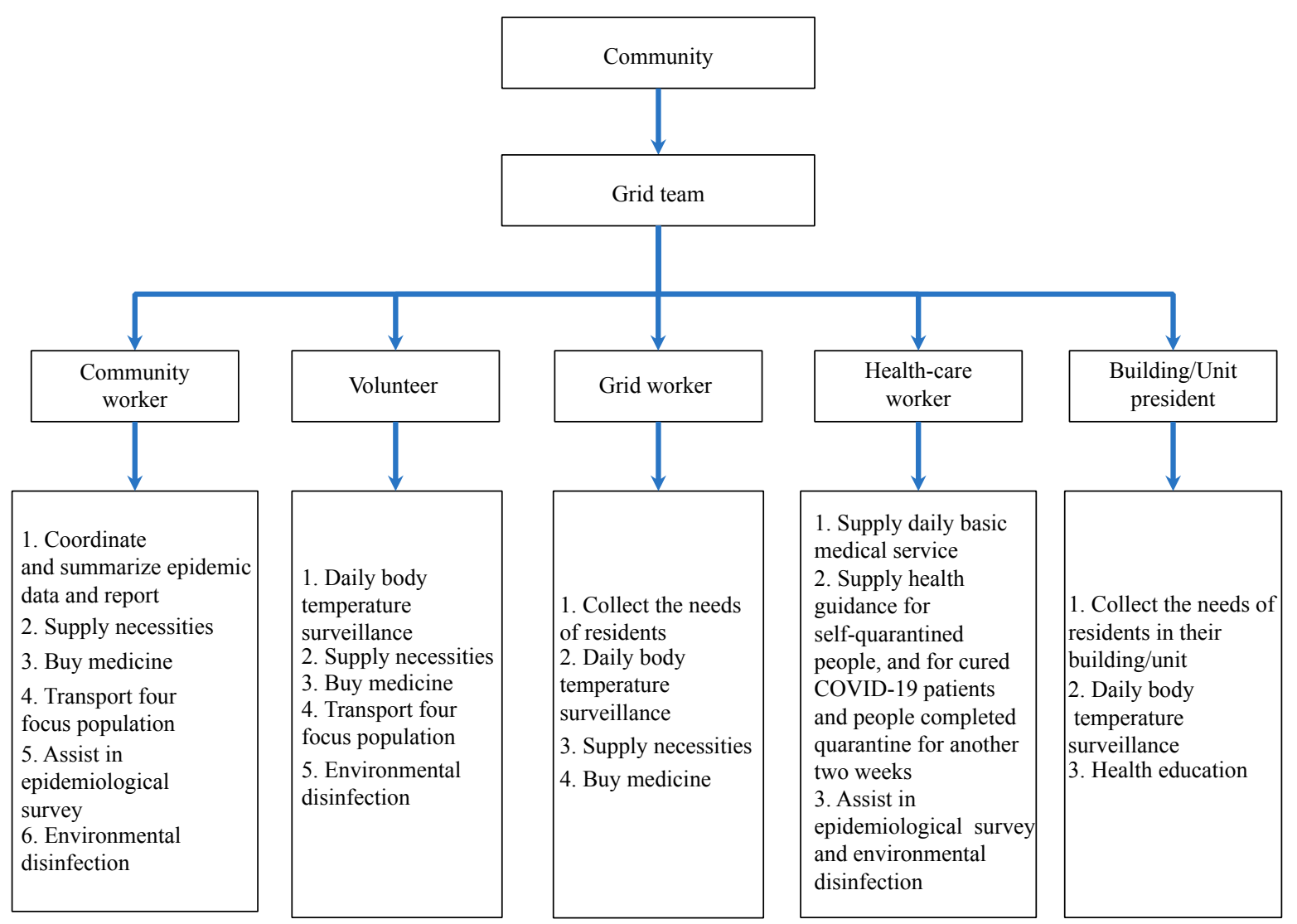

FIGURE 1. Grid team and responsibility division in Wuhan municipality.

masks and household disinfectants. Body temperature measurements at the entrance and other measures also contributed to the COVID-19 case finding and reduced the risk of transmission.

Necessities supply for residents: To reduce direct contact transmission of COVID-19 and to coordinate with the call to "stay at home", supplying foods and other necessities for residents were an important mission for community workers and volunteers. The residents had a variety of food choices and could shop online individually or buy as groups from supermarket chains via community workers or proprietor group. All goods ordered would be delivered to the entrance of the neighborhood, then the community workers and volunteers informed the residents to get their goods in batches at different times, without direct contact, and while maintaining social distancing. The government also supplied donated foods and/or low price foods for vulnerable people like disabled individuals, seniors living alone, etc. Community workers and volunteers also helped patients with chronic diseases buy medicine or visit hospitals if needed.

Daily screening and specific screening: The grid workers and volunteers worked together to collect the daily body temperature of all residents through WeChat groups, Weilinli (a WeChat program), mobile phones, and smart calling systems using automated voices. Moreover, the city implemented two specific screenings between February 6-9 and February 17-19 to find 4 focus population, including diagnosed COVID-19 patients, suspected cases, patients with fever, and close contacts, and 6,326 diagnosed COVID-19 patients and suspected cases were found. The community workers or volunteers would transport these people from the community to designated treatment facilities. All diagnosed COVID-19 patients were hospitalized, all suspected COVID-19 patients, patients with fever, and close contacts were quarantined in independent areas to have further tests as soon as possible.

Management of four-focus population: At the early stages of the pandemic, many confirmed COVID-19 cases, suspected cases, patients with fever, and close contacts quarantined themselves at home. We carried out the "five for one" policy where a team with five staff members were responsible for the management of one quarantined person. The five-staff team included a street-level officer, a grid worker, a community worker, 
a HCW, and a volunteer. They were in charge of body temperature surveillance, personal protection and family disinfection education, psychological counseling, and life support for self-quarantined people whom would also receive a health package with a thermometer, a face mask, a pen, a registration form, and a set of promotional materials.

After February 17-19, all four focus population were transported out of the community for hospitalization or centralized quarantine. However, the "five for one" policy was still in place for people who came back to community from the hospitals or the quarantine zones, and they would be under medical observation for another two weeks where the community workers would transport them back to the hospital immediately if they developed any symptoms related to COVID-19.

Centralized quarantine: Based on our clinical experience, about $80 \%$ of the COVID-19 patients had mild type and self-limited cases. These patients had two choices, self-quarantine at home or centralized quarantine. A total of 14 temporary hospitals reconstructed from hotels, exhibition halls, stadiums, schools, etc., were available to hospitalize mild COVID-19 patients. And all other 3 focus population were also quarantined in requisitioned hotels near where they live.

Health education and promotion: During the outbreak we also face an infodemic where rumors and misinformation were spread. To disseminate COVID19 knowledge, current control policies, and control measures, many health education and promotion programs were broadcast via television every day. The major information included wearing a face mask, hand hygiene, social distancing, natural ventilation, cough etiquette, etc. Communities displayed posters, distributed paper promotional materials in high visibility places, and broadcast to the community regularly. Some communities even used drones to broadcast this information.

Environmental disinfection and epidemiological survey: The local CDC staff carried out professional terminal disinfection and epidemiological surveys when a newly diagnosed COVID-19 case emerged. At the same time, local CDC would train the HCWs from Community Health Service Center, the staff of property management, and grid workers to assist local CDC staff in finishing epidemiological surveys, reconfirming some critical information of the case and his/her close contacts, and conducting daily environmental disinfection in the community including elevators, corridors, stairways of the building, door handles, public outdoor fitness equipment, waste containers, etc.

\section{Information Sharing Mechanism}

We also recommended building an information sharing mechanism among the communities, community health service center, and local CDC so that information on newly diagnosed COVID-19 cases, suspected cases, patients with fever, and close contacts could be shared promptly and would help recognize high risk populations and decrease delays for hospitalization and quarantine.

\section{Supervision and Consultation}

We, the community work group, are supervisors of community-level control and also the consultants of policy makers in Wuhan. We developed control guidelines used by communities, Community Health Service Centers, property management companies, and volunteers. The community work group also offered advice to policymakers of different levels based on evidence obtained in the communities. This advice could be transferred into policy or control measures rapidly by different level of governments.

\section{Conclusion}

Measures were focused in the community level with professional epidemiologists as supervisors. All these measures, based on the major transmission mode of COVID-19-droplet transmission and contact transmission-reduce the mobility and contact of people and disrupt transmission routes to reduce $\mathrm{R}_{0}$ quickly. Meanwhile, guaranteeing living supplies and medication requirements of chronic disease patients are key to allowing residents to stay at home. We believe this can be shared all over the world to tackle the current pandemic.

Contributors: HW, GFG, and CZ conceived and designed the framework. CZ, HW, and GFG reviewed the literature and wrote the first draft. CZ drew the figures. HW, GFG, and ZW reviewed and revised the manuscript. All authors approved the final version.

Acknowledgments: We thank all the members of the community work team .

Conflict of interests: No conflict of interests were reported.

doi: $10.46234 / \mathrm{ccdcw} 2020.126$

\# Corresponding author: Hao Wu, wushunzhe@sohu.com.

${ }^{1}$ Chinese Center for Disease Control and Prevention, Beijing, China;
${ }^{2}$ Department of Health Policy and Management, School of Public 
Health, Peking University, Beijing, China; ${ }^{3}$ Fangzhuang Community Health Service Center of Fengtai District, Capital Medical University, Beijing, China.

Submitted: June 11, 2020; Accepted: June 14, 2020

\section{REFERENCES}

1. Wang C, Horby PW, Hayden FG, Gao GF. A novel coronavirus outbreak of global health concern. Lancet 2020;395(10223):470 - 3 . http://dx.doi.org/10.1016/S0140-6736(20)30185-9.

2. Zhu N, Zhang DY, Wang WL, Li XW, Yang B, Song JD, et al. A novel coronavirus from patients with pneumonia in China, 2019. N Engl J Med 2020;382:727 - 33. http://dx.doi.org/10.1056/NEJMoa2001017.
3. The 2019-nCoV Outbreak Joint Field Epidemiology Investigation Team, Li Q. An outbreak of NCIP (2019-nCoV) infection in China Wuhan, Hubei province, 2019-2020. China CDC Weekly 2020;2(5):79 - 80. http://dx.doi.org/10.46234/ccdcw2020.022.

4. Li Q, Guan XH, Wu P, Wang XY, Zhou L, Tong YQ, et al. Early transmission dynamics in Wuhan, China, of novel coronavirus-infected pneumonia. N Engl J Med 2020;382:1199 - 207. http://dx.doi.org/ 10.1056/NEJMoa2001316.

5. World Health Organization. Coronavirus disease 2019 (COVID-19) situation report-51. https:/www.who.int/docs/default-source/coronavir use/situation-reports/20200311-sitrep-51-covid-19.pdf?sfvrsn=1ba62e57_ 10. [2020-04-22].

6. Li ZJ, Chen QL, Feng LZ, Rodewald L, Xia YY, Yu HL, et al. Active case finding with case management: the key to tackling the COVID-19 pandemic. Lancet 2020. http://dx.doi.org/10.1016/S0140-6736(20) 31278-2. 